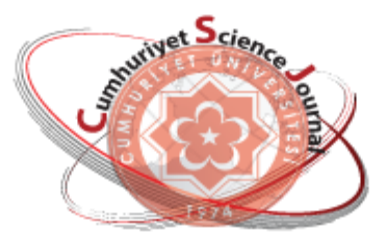

e-ISSN: $2587-246 X$

ISSN: $2587-2680$

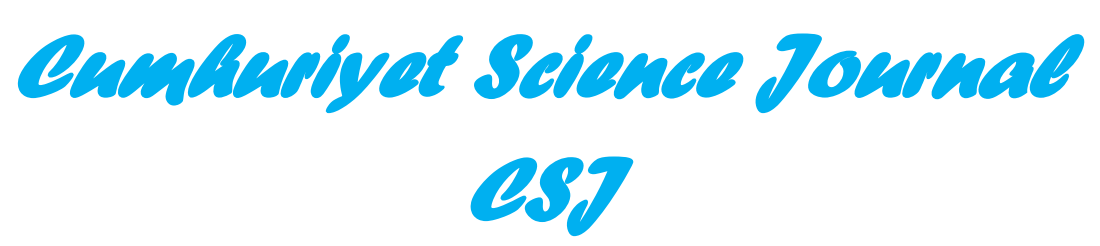

Cumhuriyet Sci. J., Vol.40-4 (2019) 784-791

\title{
The Regularized Trace Formula Of A Second Order Differential Equation Given With Anti-Perıdic Boundary Conditions
}

\author{
Seda KIZILBUDAK ÇALIŞKAN ${ }^{1}$ (iD) Leyla ÖZEN ${ }^{1}$ \\ ${ }^{1}$ Yıldiz Technical University, Faculty of Arts and Science, Department of Mathematics, (34210), Davutpaşa, \\ Istanbul,TURKEY \\ Received: 09.11.2018; Accepted: 24.10.2019 \\ http://dx.doi.org/10.17776/csj.480810
}

Abstract. In this study, we examined the formula of the regularized trace of the self-adjoint operator which is formed by

$\ell(y)=-y^{\prime \prime}+p(x) y$

differential expression and

$y(0)+y(\pi)=0$

$y^{\prime}(0)+y^{\prime}(\pi)=0$

anti-periodic boundary condition.

Keywords: Regularized trace, Eigenvalues, Eigen functions.

\section{Ters Periyodik Sınır Koşulları İle Verilmiş İkinci Mertebeden Diferansiyel Denklemin Düzenli İz Formülü}

Özet. Bu çalışmada,

$\ell(y)=-y^{\prime \prime}+p(x) y$

diferansiyel ifadesi ve

$y(0)+y(\pi)=0$

$y^{\prime}(0)+y^{\prime}(\pi)=0$

ters periyodik sınır koşulları ile oluşturulmuş kendine eş operatörün düzenli iz formülü incelenmiştir.

Anahtar Kelimeler: Düzenli iz, Öz değer, Öz fonksiyon.

\section{INTRODUCTION}

$p(x)$ is a real valued, continuous function in $[0, \pi], L_{0}$ and $L$ get two self-adjoint operators generated by the following expressions

$$
\ell_{0}(y)=-y^{\prime \prime}
$$

and

$$
\ell(y)=-y^{\prime \prime}+p(x) y
$$


with the same boundary conditions

$$
\begin{gathered}
y(0)+y(\pi)=0 \\
y^{\prime}(0)+y^{\prime}(\pi)=0
\end{gathered}
$$

respectively, in the space $L_{2}[0, \pi]$. The spectrum of operator $L_{0}$ coincides with the set $\left\{(2 n+1)^{2}\right\}_{n=0}^{\infty}$. Every point of the spectrum is an eigenvalue with multiplicity two.

Let

$$
\mu_{k}=\left\{\begin{aligned}
k^{2}, \text { if } k \text { is odd } \\
(k-1)^{2}, \text { if } k \text { is even }
\end{aligned} \quad(k=1,2, \ldots)\right.
$$

is the eigenvalues of operator $L_{0}$ and

$$
\psi_{1}=\sqrt{\frac{2}{\pi}} \sin x, \psi_{2}=\sqrt{\frac{2}{\pi}} \cos x, \psi_{3}=\sqrt{\frac{2}{\pi}} \sin 3 x, \psi_{4}=\sqrt{\frac{2}{\pi}} \cos 3 x, \ldots
$$

are the orthonormal eigenfunctions corresponding to this eigenvalues.

Also we showed the eigenvalues of operator $L$ by $\lambda_{1} \leq \lambda_{2} \leq \lambda_{3} \leq \cdots \leq \lambda_{k} \leq \cdots$ and corresponding orthonormal eigenfunctions by $\varphi_{0}, \varphi_{1}, \varphi_{2}, \ldots, \varphi_{k}, \ldots$

In this study, we obtained a formula for the sum of series by Dikii's method,

$$
\sum_{n=1}^{\infty}\left(\lambda_{n}-\mu_{n}\right)
$$

which is called the formula of regularized trace of operator $L$.

The regularized trace theory, which was first examined by Gelfand and Levitan and they derived the formula of regularized trace for the Sturm-Liouville operator [1], attracted the attention of many authors. Dikii [2] provided and developed Gelfand and Levitan's formulas by their own method. Later, Levitan [6] suggested one more method for computing the traces of the Sturm-Liouville operator. There are numerous investigations on the calculation of the regularized trace of differential operator equations [3-17].

\section{CALCULATION}

Let us show the following equation

$$
\lim _{N \rightarrow \infty} \sum_{n=1}^{N}\left[\left(\varphi_{n}, L \varphi_{n}\right)-\left(\psi_{n}, L \psi_{n}\right)\right]=0
$$

which will be used later. For this we consider the transfer matrix $\left(u_{i k}\right)_{i, k=1}^{\infty}$ from the orthonormal basis $\left\{\varphi_{k}\right\}$ to orthonormal basis $\left\{\psi_{k}\right\}$ as in [2] :

$$
\psi_{k}=\sum_{i=1}^{\infty} u_{i k} \varphi_{i} \quad(k=1,2, \ldots)
$$


where $u_{i k}=\left(\varphi_{i}, \psi_{k}\right)$ and $\left(u_{i k}\right)_{i, k=1}^{\infty}$ are the unitary matrix, that is

$$
\sum_{i=1}^{\infty} u_{i k}^{2}=1 \quad(k=1,2, \ldots)
$$

Let us give some limitations for $u_{i k}$. It is clear that

$$
L \psi_{k}=\mu_{k} \psi_{k}+p \psi_{k}
$$

If we multiply both side of equality (4) by $\varphi_{i}$ we obtain

$$
\left(L \psi_{k}, \varphi_{i}\right)=\left(\mu_{k} \psi_{k}, \varphi_{i}\right)+\left(p \psi_{k}, \varphi_{i}\right)
$$

Or

and

$$
\lambda_{i}\left(\psi_{k}, \varphi_{i}\right)=\mu_{k}\left(\psi_{k}, \varphi_{i}\right)+\left(p \psi_{k}, \varphi_{i}\right)
$$

$$
\left(\lambda_{i}-\mu_{k}\right)\left(\psi_{k}, \varphi_{i}\right)=\left(p \psi_{k}, \varphi_{i}\right)
$$

With respect to [2] taking the square of both sides of the last equality and summing from 1 to $\infty$ respect to $i$ we obtain

$$
\sum_{i=1}^{\infty}\left(\lambda_{i}-\mu_{k}\right)^{2}\left(\psi_{k}, \varphi_{i}\right)^{2}=\sum_{i=1}^{\infty}\left(p \psi_{k}, \varphi_{i}\right)=\left\|p \psi_{k}\right\|^{2}=\int_{0}^{\pi}\left[p(x) \psi_{k}(x)\right]^{2} d x \leq p_{0}^{2}
$$

where $p_{0}=\max _{0 \leq x \leq \pi}|p(x)|$.

Suppose that the following conditions hold:

1. For the eigenvalues and the eigenfunctions of the $L$ operator holds the asymptotic formulas

$$
\lambda_{k}=\mu_{k}+O\left(\frac{1}{k}\right), \varphi_{k}=\psi_{k}+O\left(\frac{1}{k}\right)
$$

2. $\int_{0}^{\pi} p(x) d x=0$.

Hence

$$
\sum_{i=N+1}^{\infty}\left(\lambda_{i}-\mu_{k}\right)^{2} u_{i k}^{2}<C \quad(C=\text { const. })(k<N) .
$$

We will use condition 1 in the inequalities we will obtain.

Obviously,

$$
\begin{aligned}
\sum_{i=N+1}^{\infty}\left(\lambda_{i}-\mu_{k}\right) u_{i k}^{2}<C & \Rightarrow \sum_{i=N+1}^{\infty}\left(\lambda_{i}-\mu_{k}\right)\left(\lambda_{i}-\lambda_{k}\right) u_{i k}^{2}<C \\
& \Rightarrow \sum_{i=N+1}^{\infty}\left(\lambda_{i}-\lambda_{k}\right)^{2} u_{i k}^{2}<C
\end{aligned}
$$


is obtained for all integer $N$ from equation (6)

And we obtain

$$
\sum_{i=N+1}^{\infty}\left(\lambda_{i}-\lambda_{k}\right) u_{i k}^{2} \leq \frac{C}{\lambda_{N+1}-\mu_{k}} \quad(k<N)
$$

Now let us prove the equation (3).

$$
\left(\psi_{k}, L \psi_{k}\right)=\left(\sum_{i=1}^{\infty} u_{i k} \varphi_{i}, \sum_{i=1}^{\infty} \lambda_{i} u_{i k} \varphi_{i}\right)=\sum_{i=1}^{\infty} \lambda_{i} u_{i k}^{2}
$$

If we take the sum on $\mathrm{k}$ from 1 to $N$ on both sides of this equation we get

$$
\sum_{k=1}^{N}\left(\psi_{k}, L \psi_{k}\right)=\sum_{k=1}^{N} \sum_{i=1}^{\infty} \lambda_{i} u_{i k}^{2}
$$

Since $\sum_{i=1}^{\infty} u_{k i}^{2}=1$ we get

$$
\sum_{k=1}^{N}\left(\varphi_{k}, L \varphi_{k}\right)=\sum_{k=1}^{N} \lambda_{k}=\sum_{k=1}^{N} \sum_{i=1}^{\infty} \lambda_{k} u_{k i}^{2}
$$

So now we need to prove

$$
\begin{aligned}
& \lim _{N \rightarrow \infty}\left(\sum_{k=1}^{N} \sum_{i=1}^{\infty} \lambda_{i} u_{i k}^{2}-\sum_{k=1}^{N} \sum_{i=1}^{\infty} \lambda_{k} u_{k i}^{2}\right)=0 . \\
& \sum_{k=1}^{N} \sum_{i=1}^{\infty} \lambda_{i} u_{i k}^{2}-\sum_{k=1}^{N} \sum_{i=1}^{\infty} \lambda_{k} u_{k i}^{2}=\sum_{k=1}^{N} \sum_{i=N+1}^{\infty}\left(\lambda_{i}-\lambda_{k}\right) u_{i k}^{2}+\sum_{k=1}^{N} \sum_{i=N+1}^{\infty} \lambda_{k}\left(u_{i k}^{2}-u_{k i}^{2}\right) .
\end{aligned}
$$

Let us calculate first sum on the right side of equality (9). For convenience while let $N+1$ be even number then we have

$\sum_{k=1}^{N} \sum_{i=N+1}^{\infty}\left(\lambda_{i}-\lambda_{k}\right) u_{i k}^{2}=\sum_{k=1}^{N-1} \sum_{i=N+1}^{\infty}\left(\lambda_{i}-\lambda_{k}\right) u_{i k}^{2}+\left(\lambda_{N+1}-\lambda_{N}\right) u_{(N+1) N}^{2}+\sum_{i=N+2}^{\infty}\left(\lambda_{i}-\lambda_{N}\right) u_{i N}^{2}$

Let us calculate first and third sum on the right side of equality (10) by inequality (7), for $N \rightarrow \infty$

$$
\sum_{k=1}^{N} \sum_{i=N+1}^{\infty}\left(\lambda_{i}-\lambda_{k}\right) u_{i k}^{2}<\frac{1}{4 N}+\frac{1}{2(N+1)}\left[\ln \frac{N^{2}+N}{N-1}\right] \rightarrow 0
$$

and 


$$
\sum_{i=N+2}^{\infty}\left(\lambda_{i}-\lambda_{N}\right) u_{i N}^{2} \leq \frac{C}{\lambda_{N+2}-\mu_{N}} \leq \frac{C}{4 N+4} \rightarrow 0
$$

Now we shall calculate the second term on the right side of equality (10) when $N \rightarrow \infty$. Suppose that $N+1$ is even, we have

$$
\left(\lambda_{N+1}-\lambda_{N}\right) u_{(N+1) N}^{2} \leq N^{2}+O\left(\frac{1}{N+1}\right)-N^{2}-O\left(\frac{1}{N}\right) \rightarrow 0 \quad(N \rightarrow \infty)
$$

In this way, for even number $N+1$ from the expressions (10), (11), (12) and (13) we have

$$
\lim _{N \rightarrow \infty} \sum_{k=1}^{N} \sum_{i=N+1}^{\infty}\left(\lambda_{i}-\lambda_{k}\right) u_{i k}^{2}=0
$$

Formula (14) can also calculated for odd number $N+1$.

Now we shall calculate second sum on the right side of equality (9).

$$
u_{i k}+u_{k i}=\left(\varphi_{i}, \psi_{k}\right)+\left(\varphi_{k}, \psi_{i}\right)=-\left(\varphi_{i}-\psi_{i}, \varphi_{k}-\psi_{k}\right)
$$

By equality (15) and condition 1., we have

$$
\left|u_{i k}+u_{k i}\right| \leq\left\|\varphi_{i}-\psi_{i}\right\|\left\|\varphi_{k}-\psi_{k}\right\|<\frac{C}{i k} .
$$

According to Cauchy-Schwarz inequality we have

$$
\begin{aligned}
\sum_{i=N+1}^{\infty}\left(\lambda_{i}-\mu_{k}\right)\left|u_{i k}^{2}-u_{k i}^{2}\right| & =\sum_{i=N+1}^{\infty}\left(\lambda_{i}-\mu_{k}\right)\left|u_{i k}-u_{k i}\right|\left|u_{i k}+u_{k i}\right| \\
& \leq \sqrt{\sum_{i=N+1}^{\infty}\left|u_{i k}-u_{k i}\right|^{2}} \sqrt{\sum_{i=N+1}^{\infty}\left(\lambda_{i}-\mu_{k}\right)^{2}\left|u_{i k}-u_{k i}\right|^{2}} \\
& <\frac{C}{(k-1) \sqrt{N+1}} .
\end{aligned}
$$

Hence

$$
\sum_{i=N+1}^{\infty}\left|u_{i k}^{2}-u_{k i}^{2}\right|<\frac{C}{(k-1) \sqrt{N+1}\left[N^{2}-(k-1)^{2}\right]}
$$

Now we shall evaluate the second sum on the right side of equality (9),

$$
\sum_{k=1}^{N} \lambda_{k} \sum_{i=N+1}^{\infty}\left|u_{i k}^{2}-u_{k i}^{2}\right|=\lambda_{N} \sum_{i=N+1}^{\infty}\left|u_{i N}^{2}-u_{N i}^{2}\right|+\sum_{k=1}^{N-1} \lambda_{k} \sum_{i=N+1}^{\infty}\left|u_{i k}^{2}-u_{k i}^{2}\right|
$$




$$
=\lambda_{N}\left|u_{N+1 N}^{2}-u_{N N+1}^{2}\right|+\lambda_{N} \sum_{i=N+2}^{\infty}\left|u_{i N}^{2}-u_{N i}^{2}\right|+\sum_{k=1}^{N-1} \lambda_{k} \sum_{i=N+1}^{\infty}\left|u_{i k}^{2}-u_{k i}^{2}\right|
$$

By inequality (16) we have

$$
\begin{aligned}
& \lambda_{N}\left|u_{N+1 N}^{2}-u_{N N+1}^{2}\right|=\lambda_{N}\left|u_{N+1 N}-u_{N N+1}\right|\left|u_{N+1 N}+u_{N N+1}\right| \\
&<\frac{C N^{2}}{N^{2}(N+1)^{2}}\left|u_{N+1 N}-u_{N N+1}\right| \rightarrow 0 \quad(N \rightarrow \infty)
\end{aligned}
$$

By the expression (18) we evaluate the second and third sum on the right side of equality (19)

$$
\lambda_{N} \sum_{i=N+2}^{\infty}\left|u_{i N}^{2}-u_{N i}^{2}\right|<\frac{C N^{2}}{(N-1) \sqrt{N+2}\left[(N+2)^{2}-(N+1)^{2}\right]} \rightarrow \infty(N \rightarrow \infty)
$$

and

$$
\sum_{k=1}^{N-1} \lambda_{k} \sum_{i=N+1}^{\infty}\left|u_{i k}^{2}-u_{k i}^{2}\right|<\frac{C N}{\sqrt{N+1}} \sum_{k=2}^{N} \frac{1}{N^{2}-(k-1)^{2}} \sim C \frac{\ln N}{\sqrt{N}} \rightarrow 0(N \rightarrow \infty) .
$$

From the expressions (19), (20),(21) and (22) we have

$$
\lim _{N \rightarrow \infty} \sum_{k=1}^{N} \sum_{i=N+1}^{\infty} \lambda_{k}\left(u_{i k}^{2}-u_{k i}^{2}\right)=0
$$

Thus from the expressions (9), (14), and (23) we obtain formula (8). Therefore formula (3) have proved.

\section{CONCLUSION}

$$
\left(\varphi_{k}, L \varphi_{k}\right)=\lambda_{k} \quad \text { and } \quad\left(\psi_{k}, L \psi_{k}\right)=\mu_{k}+\left(\psi_{k}, p \psi_{k}\right)
$$

If we use these into formula (3) then we obtain

$$
\sum_{k=1}^{N}\left[\left(\psi_{k}, L \psi_{k}\right)-\left(\varphi_{k}, L \varphi_{k}\right)\right]=\sum_{k=1}^{N}\left(\mu_{k}-\lambda_{k}\right)+\sum_{k=1}^{N}\left(\psi_{k}, p \psi_{k}\right) \rightarrow 0, \quad(N \rightarrow \infty) .
$$

Now we shall calculate

$$
\lim _{N \rightarrow \infty} \sum_{k=1}^{N}\left(\psi_{k}, p \psi_{k}\right)
$$

According to condition 2. we have for even number $N$ 


$$
\sum_{k=1}^{N}\left(\psi_{k}, p \psi_{k}\right)=\frac{1}{\pi} \int_{0}^{\pi} p(x) d x+\frac{N}{\pi} \int_{0}^{\pi} p(x) d x=0
$$

Similarly we have for odd number $\mathrm{N}$

$$
\sum_{k=1}^{N}\left(\psi_{k}, p \psi_{k}\right)=-\frac{1}{\pi} \int_{0}^{\pi} p(x) \cos 2 N x d x \rightarrow 0, \quad(N \rightarrow \infty) .
$$

From the expressions (25) and (26) we have

$$
\lim _{N \rightarrow \infty} \sum_{k=1}^{N}\left(\psi_{k}, p \psi_{k}\right)=0
$$

Hence from the expressions (24) and (26) we have

So we have proved the following theorem.

$$
\lim _{N \rightarrow \infty} \sum_{k=1}^{N}\left(\lambda_{k}-\mu_{k}\right)=0
$$

THEOREM : The following formula is true when we considered $p(x)$ is a continuous function and conditions $1 ., 2$. are fulfilled

$$
\sum_{n=1}^{\infty}\left(\lambda_{n}-\mu_{n}\right)=0
$$

\section{REFERENCES}

[1] Gelfand, I. M. and Levitan, B. M., On a formula for eigenvalues of a differential operator of second order. Dokl. Akad. Nauk SSSR, 88-4 (1953) 593-596.

[2] Dikii, L. A., On a Formula of Gelfand-Levitan. Usp. Mat. Nauk, 8-2 (1953) 119-123.

[3] Gelfand, I. M., About an identity for eigenvalues of a differential operator of second order. Usp. Mat. Nauk, 11(67) (1956) 191-198.

[4] Fadeev, L.D., On the Expression for the Trace of the Difference of Two Singular Differential Operators of the Sturm-Liouville Type. Dokl. Akad. Nauk SSSR, 115-5 (1957) 878881.

[5] Dikii, L. A., Trace formulas for differential operators of Sturm- Liouville. Uspeki Matem. Nauk, 13-3 (1958) 111-143.

[6] Levitan, B.M., Calculation of the Regularized Trace for the Sturm-Liouville Operator. Uspekhi Mat. Nauk, 19-1 (1964) 161-165.

[7] Sadovnichii,V.A.,On the trace of the difference of two high-order ordinary differential operators. Differents, Uravneniya, 2-12 (1966) 1611-1624.

[8] Cao, C.W. and Zhuang, D.W., Some trace formulas for the Schr"odinger equation with energy-dependent potential, Acta Math. Sci.(in Chinese), 5 (1985) 233-236. 
[9] Bayramoğlu, M., On the regularized trace formula of th differential equation with unbounded Coefficient. Spectral Theory and Its Applications, 7 (1987) 15-40.

[10] Lax P. D., Trace formulas for the Schroeding operator. Commun. Pure Appl. Math., 47- 4 (1994) 503-512.

[11] Papanicolaou, V.G., Trace formulas and the behavior of large eigenvalues, SIAM J. Math. Anal., 26 (1995), 218-237.

[12] Adıgüzelov, E. E., Baykal, O. and Bayramov, A., On the spectrum and regularized trace of the Sturm-Liouville problem with spectral parameter on the boundary condition and with the operator coefficient. International Journal of Differential Equations and Applications, 2-3 (2001) 317-333.

[13] Savchuk, A.M., Shkalikov, A.A., Trace formula for Sturm-Liouville Operators with Singular Potentials. Mathematical Notes, 69-3 (2001).

[14] Bayramov, A., Öztürk Uslu, S. and Kızılbudak Çalışkan, S., On the trace formula of second order differential equation given with non-seperable boundary conditions. Sigma Journal of Engineering and Natural Sciences, 4 (2005) 57-64.

[15] Guliyev, N.J., The regularized trace formula for the Sturm-Liouville equation with spectral parameter in the boundary condition. Proceedins of IMM of NAS of Azerbaijan, 22 (2005) 99-102.

[16] Sadovnichii, V.A. and Podol'skii, V.E., Traces of Differential Operators. Differential Equations, 45- 4 (2009) 477-493

[17] Wang, Y.P., Koyunbakan H. and Yang, C.F., A Trace Formula for Integro-differential Operators on the Finite Interval, Acta Mathematicae Applicatae Sinica (English Series) 33-1 (2017) 141-146. 